\title{
Synergistic antitumor activity of a DLL4/VEGF bispecific therapeutic antibody in combination with irinotecan in gastric cancer
}

\author{
Da-Hyun Kim ${ }^{1,2}$, Seul Lee ${ }^{1}$, Hyeok Gu Kang ${ }^{1,2}$, Hyun-Woo Park ${ }^{3}$, Han-Woong Lee ${ }^{3}$, Dongin Kim ${ }^{4}$, Dong-Hoon Yoem ${ }^{4}$, \\ Jin-Hyung Ahn ${ }^{4}$, Eunsin Ha ${ }^{4,7}$, Weon-Kyoo You ${ }^{4}$, Sang Hoon Lee ${ }^{4}$, Seok-Jun Kim ${ }^{5,6, *}$ E Kyung-Hee Chun ${ }^{1,2, *}$ \\ ${ }^{1}$ Department of Biochemistry \& Molecular Biology, Yonsei University College of Medicine, ${ }^{2}$ Brain Korea 21 Plus Project for Medical \\ Science, Yonsei University College of Medicine, ${ }^{3}$ Department of Biochemistry, College of Life Science, Yonsei University, Seoul 03722, \\ ${ }^{4} \mathrm{R} \& \mathrm{D}$ center, ABL Bio Inc., Seongnam 13488, ${ }^{5}$ Department of Biomedical Science, College of Natural Science, Chosun University, \\ ${ }^{6}$ Brain Korea 21 Plus Research Team for Bioactive Control Technology, College of Natural Sciences, Chosun University, Gwangju 61452 , \\ ${ }^{7}$ National OncoVenture, National Cancer Center, Goyang 10408, Korea
}

Notch signaling has been identified as a critical pathway in gastric cancer (GC) progression and metastasis, and inhibition of Delta-like ligand 4 (DLL4), a Notch ligand, is suggested as a potent therapeutic approach for GC. Expression of both DLL4 and vascular endothelial growth factor receptor 2 (VEGFR2) was similar in the malignant tissues of GC patients. We focused on vascular endothelial growth factor (VEGF), a known angiogenesis regulator and activator of DLL4. Here, we used ABL001, a DLL4/VEGF bispecific therapeutic antibody, and investigated its therapeutic effect in GC. Treatment with human DLL4 therapeutic antibody (anti-hDLL4) or ABL001 slightly reduced GC cell growth in monolayer culture; however, they significantly inhibited cell growth in 3D-culture, suggesting a reduction in the cancer stem cell population. Treatment with anti-hDLL4 or ABL001 also decreased GC cell migration and invasion. Moreover, the combined treatment of irinotecan with anti-hDLL4 or ABL001 showed synergistic antitumor activity. Both combination treatments further reduced cell growth in 3D-culture as well as cell invasion. Interestingly, the combination treatment of ABL001 with irinotecan synergistically reduced the GC burden in both xenograft and orthotopic mouse models. Collectively, DLL4 inhibition significantly decreased cell motility and stem-like phenotype and the combination treatment of DLL4/VEGF bispecific therapeutic antibody with irino-

*Corresponding authors. Seok-Jun Kim, Tel: +82-62-230-6664; Fax: +82-62-234-4326; E-mail: heaven1472@chosun.ac.kr; Kyung-Hee Chun, Tel: +82-2-2228-1699; Fax: +82-2-312-5041; E-mail: khchun @yuhs.ac

https://doi.org/10.5483/BMBRep.2020.53.10.103

Received 13 May 2020, Revised 14 May 2020, Accepted 27 May 2020

Keywords: DLL4/VEGF bispecific therapeutic antibody, Gastric cancer, Irinotecan, Synergistic antitumor effect tecan synergistically reduced the GC burden in mouse models. Our data suggest that ABL001 potentially represents a potent agent in GC therapy. Further biochemical and pre-clinical studies are needed for its application in the clinic. [BMB Reports 2020; 53(10): 533-538]

\section{INTRODUCTION}

Gastric cancer (GC) is one of the most common malignancies, accounting for more than one million newly diagnosed cases each year worldwide $(1,2)$. Although the key molecular signaling pathways in GC have been researched extensively, the molecules associated with GC have not been identified (3). A few molecular-guided targeted therapies have been developed and are being widely used in the clinical treatment of patients with advanced-stage of GC; however, the overall survival remains poor (4). Therefore, further research into the molecular mechanisms in GC and novel anti-cancer drugs are needed for effective molecular-targeted therapy.

The Notch signaling pathway has been implicated in cancer cell survival, metastasis, drug resistance through the maintenance of cancer stem cells (CSCs), epithelial-mesenchymal transition (EMT), and genomic instability $(5,6)$. In mammals, there are four Notch homologous receptors (Notch 1-4), which can bind various ligands-Delta-like ligands (DLL1, 3, and 4), and Jagged 1 and 2 (7). DLL4, one of the major factors involved in Notch signaling, was shown to be responsible for tumor formation, EMT, and self-renewal as CSCs (8). Targeting DLL4 expression may be a feasible approach to interfere with angiogenesis, tumorigenesis, and metastasis for anti-cancer effect $(9,10)$. However, the clinical significance of DLL4 expression in GC is associated with poor prognosis (9) but mechanistic details remain unresolved (11).

Here, we analyzed the therapeutic potential of DLL4 inhibition using an anti-human DLL4 therapeutic antibody and ABL001, 
a DLL4/VEGF bispecific therapeutic antibody in GC. The ABL001 bispecific antibody consists of an anti-VEGF antibody (bevacizumablike) backbone C-terminally linked with a DLL4-targeting singlechain variable fragment which shows potent binding affinity to VEGF (12), suggesting that it may be a novel therapeutic antibody for cancer treatment. We also assessed the synergistic therapeutic effect of the combination treatment of ABL001 and irinotecan on GC using both xenograft and orthotopic mouse models. Irinotecan is a key chemotherapeutic drug in several cancers (13) and mediates its effect by inducing DNA damage and cell death through topoisomerase 1 activity inhibition. Recently, irinotecan has been used for advanced GC treatment as single agent (14) and in combination with 5-FU $(15,16)$. Therefore, we focused on the synergistic effect of combination treatment of irinotecan and ABL001 in GC.

\section{RESULTS}

\section{DLL4 and VEGFR2 expression and their association with survival of GC patients}

DLL4 and VEGFR2 expression in malignant stomach tissues of GC patients was obtained from the cBioPortal database (Fig. 1A). Moreover, we performed Kaplan-Meier analysis to generate a survival curve of the analyzed gene sets (low and high expression groups of both DLL4 and VEGFR2) of GC patients (Fig. 1B). Statistical analysis showed that high DLL4 and VEGFR2 expression correlated with lower survival. Therefore, we hypothesized that high DLL4 and VEGFR2 expression was related to GC progression.

A

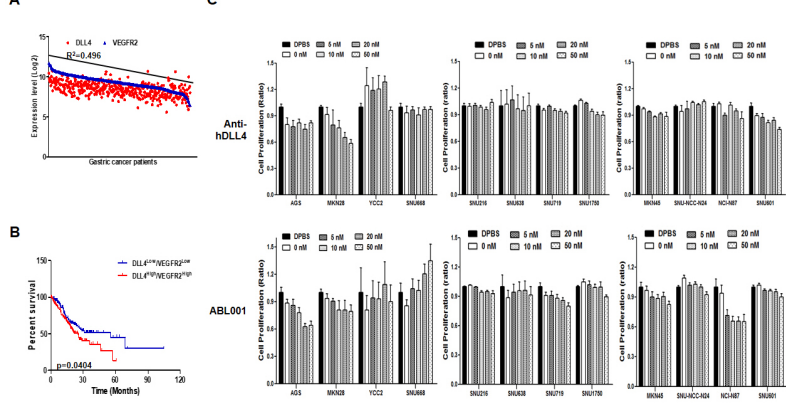

Fig. 1. DLL4 and VEGFR2 expression and the associated survival rate in GC patients, and viability of GC cell lines following treatment with anti-human DLL4 or ABL001 antibody. (A) DLL4 and VEGFR2 expression was analyzed in the malignant tissues of GC patients from the cBioPortal database. (B) Kaplan-Meier survival plots demonstrating poor prognostic value of DLL4 and VEGFR2 up-regulation, which correlates with a worse overall survival of GC patients. (C) Twelve GC cell lines were treated with different concentrations of anti-hDLL4 and ABL001 for $24 \mathrm{~h}$. Cell viability was then analyzed using WST assay and the assays were performed in triplicate.

\section{Growth inhibition of GC cell lines following treatment with anti-human DLL4 or ABL001}

We treated twelve GC cell lines with the indicated doses (Fig. 1C) of anti-human DLL4 (anti-hDLL4) and bispecific DLL4/ VEGF therapeutic antibody (ABL001) for 3 days. Anti-DLL4 mediated dose-dependent growth inhibition of AGS and MKN28 GC cells. ABL001 also mediated dose-dependent growth inhibition of AGS, MKN28, and NCl-N87 GC cells. We determined the expression of Notch 1 and 2, DLL4, Hes1, and VEGFR2 at the mRNA level in the twelve GC cell lines (Supplementary Fig. 1). Notch receptor 1 and 2 expression was detected in all the GC cells. AGS cells showed expression of both DLL4 and VEGFR2, whereas MKN28 cells showed high DLL4 expression with low VEGFR2 expression, suggesting that AGS cells were sensitive to both anti-hDLL4 and ABL001, while MKN28 cells were sensitive to anti-hDLL4. Therefore, we selected AGS cells, which were the most sensitive to both anti-hDLL4 and ABL001, and MKN28 cells, which were the most sensitive to anti-hDLL4 for further studies.

Cell cycle inhibition and apoptosis induction in GC cell lines following treatment with anti-human DLL4 or ABL001

We choose AGS and MKN28 GC cells to assess the cell cycle status following treatment with $50 \mathrm{nM}$ anti-hDLL4 or ABL001 for $24 \mathrm{hr}$ to analysis cell cycle population (Fig. 2A). Both the antibodies inhibited cell growth, but did not affect the cell cycle arrest in either AGS or MKN28 cells on day 1. Apoptosis was assessed following $24 \mathrm{~h}$ treatment with $50 \mathrm{nM}$ anti-hDLL4 or ABL001. Neither of the cell lines showed apoptosis induction following treatment (Fig. 2B). These data suggested that neither anti-hDLL4 nor ABL001 directly affects cell cycle and apoptosis of the GC cells.

Cell invasion, migration, and stem-like phenotype of GC cell lines following treatment with anti-human DLL4 or ABL001 We treated AGS and MKN28 cells with $50 \mathrm{nM}$ anti-hDLL4 or ABL001 and assessed cell motility (Fig. 2C and 2D) and stemness (Fig. 2E). Treatment of the cells with anti-hDLL4 and ABL 001 for $1 \mathrm{~d}$ significantly decreased invasion (Fig. 2C) and migration (Fig. 2D) in both the cell lines. The decrease in migration and invasion was greater in AGS than in MKN28 cells. Moreover, in 3D-culture, AGS and MKN28 cells were treated with anti-hDLL4 and ABL001 for 15 days (Fig. 2D). The number and size of the spheres were markedly reduced following treatment with anti-hDLL4 and ABL001 in both the cells, suggesting that anti-hDLL4 and ABL001 treatment decreased stem-like phenotypic character of the GC cells. Further, the decrease in the motility and stem-like phenotype was higher in AGS than in MKN28 cells with both anti-hDLL4 and ABL001; however, there was no difference in the sensitivity of the two cell lines to anti-hDLL4 and ABL001 in an in vitro culture system. 


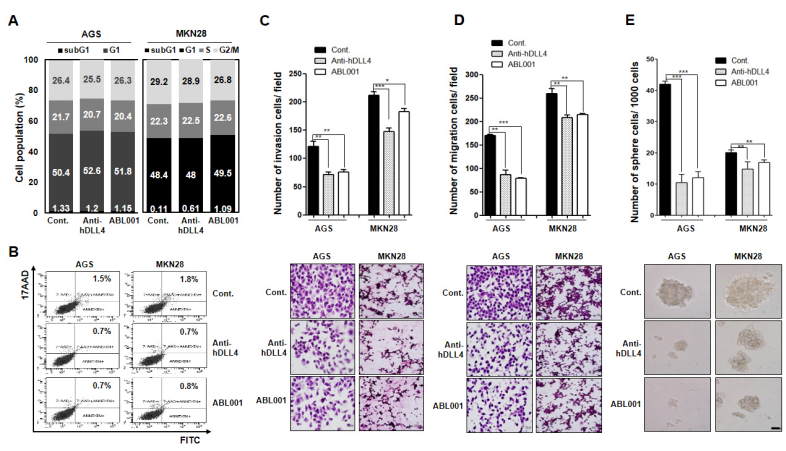

Fig. 2. Cell cycle distribution, apoptosis, cell invasion, migration, and stem-like phenotype following treatment with anti-human DLL4 or ABL001 antibody in GC cells (A, B) AGS and MKN28 GC cells were treated with $50 \mathrm{nM}$ anti-hDLL4 or ABL001 for $24 \mathrm{~h}$. (A) Cell cycle distribution was detected by PI staining and FACS analysis. (B) Apoptosis induction was detected with Annexin $\mathrm{V}$ and 17-AAD staining and FACS analysis. (C, D) AGS and MKN28 GC cells were prepared and added to Transwell chambers. (C) The upper side of the membrane was coated with collagen type I for migration assay. (D) The lower side of the membrane was coated with Matrigel for invasion assay. After incubation with $50 \mathrm{nM}$ antihDLL4 or ABL001 for $24 \mathrm{~h}$, the migrated or invaded cells in the lower chamber were quantified following H\&E staining by counting in five randomly selected areas in each well using wide-field microscopy. Data are expressed as mean \pm standard error of mean (SEM) from three independent experiments. (E) AGS and MKN28 cells $(1,000$ cells each) were grown in ultra-low attachment plates containing mammary epithelium basal medium supplemented with or without antihDLL4 or ABL001. After culturing for $15 \mathrm{~d}$, spheres with diameters of $>50 \mu \mathrm{m}$ were counted. Cells were treated every 3 days with $50 \mathrm{nM}$ concentration of the antibodies. ${ }^{*} \mathrm{P}<0.05, * * \mathrm{P}<0.01$, and $* * * \mathrm{P}<0.001$.

\section{Synergistic effect of the combination of anti-human DLL4 or ABL001 with irinotecan in GC cells}

To test the practical applicability of anti-hDLL4 and ABL001 in GC therapy, we evaluated the effect of combination treatment of anti-hDLL4, ABL001, and irinotecan in GC cells. AGS and MKN45 cells were treated with $1 \mu \mathrm{M}$ of irinotecan alone, or a combination of irinotecan $(0.5 \mu \mathrm{M})$ with anti-hDLL4 $(25 \mathrm{nM})$, or irinotecan $(0.5 \mu \mathrm{M})$ with ABL001 $(25 \mathrm{nM})$ for 2 days. Both the combination treatments showed greater inhibition of cell viability compared to treatment with irinotecan alone in AGS cells, but not in MKN28 cells (Fig. 3A). We also evaluated the inhibitory effect of the combined treatments on cell invasion. Cell invasion was slightly reduced following treatment with irinotecan alone, but the combination treatments induced significant inhibition of cell invasion (Fig. 3B).

Next, we determined the effect of the combination treatments on sphere-forming ability of AGS and MKN45 cells by performing 3D cell culture assays (Fig. 3C and 3D). Cultivation of the cells in sphere-forming culture media revealed that the size and number of spheres were smaller in both the combination treatments compared to treatment with irinotecan alone. These data suggested that both anti-hDLL4 and ABL001 show
A
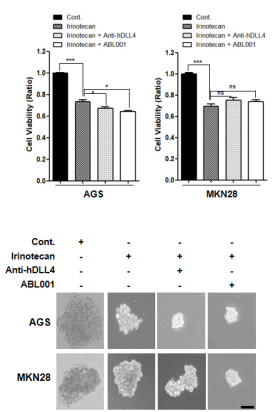

B
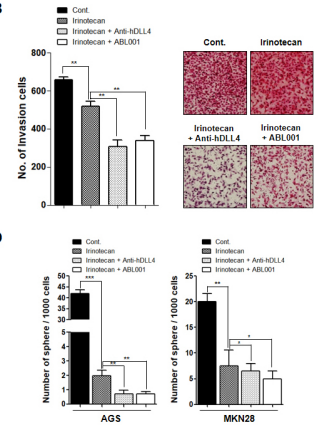

Fig. 3. Cell viability following combination treatment with irinotecan and anti-human DLL4 therapeutic antibody or irinotecan and $\mathrm{ABL}$ 001 antibody in GC cells (A, B) AGS and MKN28 cells were treated with $1 \mu \mathrm{M}$ irinotecan alone, a combination of irinotecan $(0.5$ $\mu \mathrm{M})$ and anti-hDLL4 $(25 \mathrm{nM})$, or irinotecan $(0.5 \mu \mathrm{M})$ and ABL001 $(25 \mathrm{nM})$ for $24 \mathrm{~h}$. (A) WST assays were performed to detect the cell viability. (B) Invasion ability of the cells was analyzed using Transwell chamber assay. (C, D) AGS and MKN28 cells were grown under 3D-culture conditions and the sphere-forming ability was measured after 15-days of treatment. Cells were treated with $1 \mu \mathrm{M}$ irinotecan alone, a combination of irinotecan $(0.5 \mu \mathrm{M})$ and anti-hDLL4 $(25 \mathrm{nM})$, or irinotecan $(0.5 \mu \mathrm{M})$ and ABL001 (25 nM) every 3 days. ${ }^{*} \mathrm{P}<0.05, * * \mathrm{P}<0.01$, and $* * * \mathrm{P}<0.001$.

enhanced therapeutic effect on GC cells when used in combination with irinotecan.

\section{Synergistic effect of the combination of irinotecan and} anti-mouse surrogate ABL001 in GC-bearing mouse models Next, we sought to determine the in vivo therapeutic effect of combination treatment with mouse ABL001 (mABL001) and irinotecan in a GC mouse model. We used mouse surrogate ABL001 in the mouse cancer model, because ABL001 would affect DLL4 and VEGF in the cancer tissues of the mouse models. Palpable xenografted gastric tumors were generated using AGS cells and the mice were administered $3.25 \mathrm{mg} / \mathrm{kg}$ $\mathrm{mABL} 001,10 \mathrm{mg} / \mathrm{kg}$ irinotecan, and a combination of 3.25 $\mathrm{mg} / \mathrm{kg} \mathrm{mABL} 001$ and $10 \mathrm{mg} / \mathrm{kg}$ irinotecan through intraperitoneal (i.p.) injection twice a week (Supplementary Fig. 2). Treatment with irinotecan alone did not affect the tumor growth and the tumor burden was similar to that of the control, whereas treatment with ABL001 significantly decreased the tumor burden. Interestingly, combination treatment with irinotecan and ABL001 synergistically decreased the tumor burden more than either of the treatments alone (Fig. 4A). After the mice were euthanized, the tumors were resected, photographed (Fig. 4B), and weighed (Fig. 4C). The synergistic effect of the combination treatment with irinotecan and ABL001 is shown in Fig. 4A.

Furthermore, we used an orthotopic GC mouse model, which more closely mimics the physiology of human GCs, because the sensitivity of therapeutic effect is distinct in the different locations and organs in the body. In vivo bioluminescence 

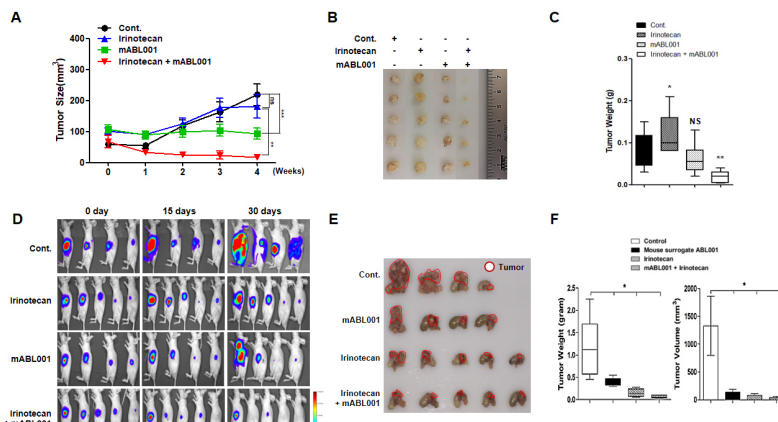

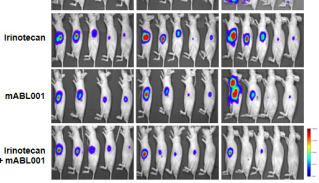

Fig. 4. Synergistic effect of combination treatment of irinotecan and anti-mouse surrogate ABL001 antibodies in GC-bearing mouse model. (A-C) AGS cells $\left(1 \times 10^{6}\right)$ were injected subcutaneously in nude mice $(n=5)$. Once palpable tumors were detectable, the mice were administrated $200 \mu \mathrm{l}$ PBS (as control), $3.25 \mathrm{mg} / \mathrm{kg} \mathrm{ABL}$ 001, $10 \mathrm{mg} / \mathrm{kg}$ irinotecan, or a combination of $3.25 \mathrm{mg} / \mathrm{kg}$ ABL001 and $10 \mathrm{mg} / \mathrm{kg}$ irinotecan by intraperitoneal (i.p.) injection twice a week. (A) Tumor size was measured every week for 5 weeks and presented graphically. (B) At the end of the treatment, the mice were euthanized and tumors were excised and photographed. (C) Tumors were weighed and the results were presented as bar graph. (D-F) Luciferase gene-transfected AGS cells were injected subcutaneously in nude mice and allowed to grow. The palpable tumor was excised and cut into sections of $10 \mathrm{~mm}^{3}$. A section of the tumor was surgically transplanted in the stomach walls of fresh nude mice $(n=5)$. After establishment of orthotopic gastric tumors, the mice were administered $200 \mu \mathrm{l} \mathrm{PBS}$ (as control), $10 \mathrm{mg} / \mathrm{kg}$ irinotecan, or a combination of $3.25 \mathrm{mg} / \mathrm{kg}$ ABL001 and $10 \mathrm{mg} / \mathrm{kg}$ irinotecan through intraperitoneal (i.p.) injection twice a week for 5 weeks. (D) In vivo bioluminescent imaging of the tumor growth in mice. (B) Mice were euthanized and the orthotopic gastric tumors were excised and photographed. (C) Tumor growth was quantified by measuring the tumor weight and volume and results were presented as graphs. ${ }^{*} \mathrm{P}<0.05,{ }^{*} \mathrm{P}<0.01$, and $* * * \mathrm{P}<0.001$.

imaging by luciferase labeling is useful in analyzing cancer cells and monitoring tumor growth (17). We generated xenograft tumors of these cells in mice. The tumors were then excised, cut into $10 \mathrm{~mm}^{3}$ sections, and surgically implanted into the stomach epithelia of the mice. After confirming that the orthotopic gastric tumors were established in the stomach of the animals, the mice were administered $3.25 \mathrm{mg} / \mathrm{kg}$ mABL001, $10 \mathrm{mg} / \mathrm{kg}$ irinotecan, and a combination of $3.25 \mathrm{mg} / \mathrm{kg} \mathrm{mABL} 001$ and $10 \mathrm{mg} / \mathrm{kg}$ irinotecan through i.p. injection twice a week for 4 weeks (Supplementary Fig. 3). The orthotopic tumor growth in mouse stomach was measured by tomographic imaging (Fig. $4 \mathrm{D})$, the orthotopic tumors were excised, photographed (Fig. 4E), and their weights and volumes were measured (Fig. 4F). The therapeutic effect of the combination treatment of irinotecan with mABL001 was greater than that of either of the single treatments or PBS treatment. The decrease in tumor weight and volume was the most significant in the combination treatment with irinotecan and mABL001 than in either of the single treatments or that observed in the xenograft mouse model described earlier. Interestingly, the treatment with irinotecan alone had better therapeutic effect in the orthotopic GC mouse model than in the xenograft GC mouse model, whereas mABL001 had consistent therapeutic effect in both the GC mouse models. These results strongly suggested that ABL001 has anticancer effect in GC mouse models and that the combination treatment of irinotecan and ABL001 has synergic anti-GC effect.

\section{DISCUSSION}

Despite surgical resection and systemic chemotherapy, advancedstage GC patients with distant metastasis continue to have poor prognosis $(18,19) .5-\mathrm{FU}$ and cisplatin are the major drug used in GC treatment and anti-cancer drugs are often combined for GC treatment (20). Irinotecan, is a key chemotherapeutic drug used in combination treatment with 5-FU for metastatic colorectal cancer (21). Irinotecan induces DNA damage and cell death through the inhibition of topoisomerase 1 activity. Recently, irinotecan has been used to treat advanced-stage GC as single treatment $(14,16)$ or in combination with other agents $(22,23)$.

In this study, we determined the inhibition of DLL4 to be a potent approach in GC therapy. We were especially interested in therapeutic effect of ABL001, a DLL4 and VEGF bispecific therapeutic antibody in GC. ABL001 competitively inhibited the binding of ligands to their corresponding receptors, including that of DLL4 to Notch1 and VEGF to VEGFR2 (12). DLL4 is a Notch ligand that regulates the activity of the Notch pathway and its expression highly correlates with tumor angiogenesis and metastasis $(10,24,25)$. VEGFR2 belongs to the receptor type tyrosine kinase gene family, and is involved in tumor angiogenesis, chemoresistance, and aorta survival (26). Currently, agents that target the VEGF (an activator of DLL4) pathway, such as bevacizumab, and inhibit angiogenesis are being used in the treatment of cancers; however, their efficacy is limited in some patients. Therefore, therapeutic strategies based on bispecific antibody targeting DLL4 and VEGF holds great promise.

We first evaluated the effect of anti-DLL4 and ABL001 treatment in twelve GC cell lines. Unexpectedly, anti-hDLL4 and ABL001 treatment could not effectively reduce the growth of majority of the GC cell lines growing as monolayers in culture dishes. Only AGS and MKN-28 cells showed a dose-dependent reduction in growth following treatment with these antibodies. However, both anti-hDLL4 and ABL001 had a significant effect on AGS and $\mathrm{MKN}-28$ cells in 3D-culture reducing sphere formation (indicative of stem-like cells). CSCs are responsible for tumor relapse, chemoresistance, and cancer metastasis $(8,27)$. Conventional chemotherapy offers limited benefits in advancedstage GC patients and prognosis for such patients remains poor. Therefore, regulation of CSCs through the development of novel agents may improve the treatment and survival of GC patients. Notch signaling triggered by the interaction of DLL4 plays a crucial role in GC stem cell pathology (28). Here, we demonstrate DLL4 inhibition using both anti-DLL4 and ABL001 significantly reduced the stem-like phenotype in GC and anti-mouse mABL001 administration significantly reduced the tumor burden 
in both xenograft and orthotopic GC-bearing mice.

Furthermore, we demonstrated the synergistic antitumor effect of combination treatment of ABL001 with irinotecan. Combined treatment of ABL001 with irinotecan synergistically reduced sphere formation of GC cell in a 3D culture compared to that by either single treatment. Further, this synergism was greater in in vivo GC-bearing mice. The combined administration of ABL001 with irinotecan reduced GC tumor burden compared to that by PBS or single agent administration alone. However, the molecular mechanisms involved in this synergistic antitumor activity remain unclear and is the topic of our current research.

Collectively, our data suggest that DLL4 inhibition reduced the stem-like phenotype of cancer cells, and cancer cell migration and invasion. ABL001, a bispecific DLL4 and VEGF therapeutic antibody may be a promising agent in GC therapy, because it showed strong antitumor effect in vivo. Moreover, the combination treatment with irinotecan and ABL001 showed synergistic antitumor activity in GC-bearing mice, suggesting that combination treatment with irinotecan and ABL001 is a novel therapeutic approach in GC. However, further studies are needed for its application in GC therapy.

\section{MATERIALS AND METHODS}

\section{Cell culture}

The human GC cell lines AGS, SCH, YCC-2, MKN28, MKN45, SNU216, SNU601, SNU638, SNU668, SNU719, SNU1750, NCC24, and N87 were obtained from KCLB (Seoul, Korea) and maintained in 1640 medium supplemented with $10 \%$ fetal bovine serum (FBS) and 1\% antibiotics (Invitrogen, Carlsbad, CA, USA) at $37^{\circ} \mathrm{C}$ in an atmosphere of $5 \% \mathrm{CO}_{2}$ as described previously $(29,30)$. Bispecific DLL4/VEGF antibody, ABL001 (NOV1501/TR009) and irinotecan were obtained from ABL Bio (Pangyo, Korea).

\section{D cell culture assays}

Cells $(1,000$ cells $/ \mathrm{ml})$ were grown in ultra-low attachment plates (Corning Costar, Acton, MA, USA) containing mammary epithelium basal medium (Lonza, Basel, Switzerland) supplemented with B27 (Gibco, Grand island, NY, USA), $20 \mathrm{ng} / \mathrm{ml}$ EGF, and $20 \mathrm{ng} / \mathrm{ml}$ bFGF (Peprotech, Rocky Hill, NJ, USA). After culturing for 15 days, spheres with diameters of $>50$ $\mu \mathrm{m}$ were counted (31).

\section{Preparation of orthotopic transplanted GC-bearing mouse models}

All animal experiments were approved by the Institutional Review Board of the Yonsei University College of Medicine (IRB number: 2018-0155). The animals were maintained in specific pathogen-free facilities and used in accordance with the University's Guidelines for the Care and Use of Laboratory Animals. Six-week-old female BALB/c nude mice (Orient, Korea) were subcutaneously inoculated with AGS luciferase cells $\left(1 \times 10^{6} \%\right.$
$100 \mu l)$ in the flank side. Subcutaneous tumors were excised and implanted in the gastric wall of nude mice. Mice were randomized into groups ( $\mathrm{n}=5 /$ group) and treatment was started 4 weeks after tumor implantation. Mice received $200 \mu \mathrm{l}$ PBS (as control), $3.25 \mathrm{mg} / \mathrm{kg}$ mouse surrogate ABL001, 10 $\mathrm{mg} / \mathrm{kg}$ irinotecan, or a combination of $3.25 \mathrm{mg} / \mathrm{kg} \mathrm{ABL001}$ and $10 \mathrm{mg} / \mathrm{kg}$ irinotecan through intraperitoneal (i.p.) injection twice a week. Once every 2 weeks, the mice were injected i.p. with luciferin (Xenogen, Alameda, CA) and luciferase activity was recorded and measured using IVIS imaging. The experiment was terminated at 5 weeks and the volumes of the orthotopically implanted tumors were calculated using the formula $a^{2} \times b \times 0.5$.

\section{Statistical analysis}

Unpaired (two sample) t test was used to determine the Pvalues. P-values $<0.05$ were considered to be statistically significant. Statistical analyses were performed using Prism 5 (GraphPad software, La Jolla, CA, USA).

\section{ACKNOWLEDGEMENTS}

This work was supported by the Bio \& Medical Technology Development Program of the NRF funded by the Korean government, MSIP (NRF-2015M3A9B6073835, NRF-2015M3 A9B6073833) and National Research Foundation of Korea (NRF) grant funded by the Korea government NRF-2017R1A 2B2006238, NRF-2019R1A2C2089237, NRF-2017R1C1B2005265 to S.J.K.

This study was supported by the National OncoVenture Program (No. HI11C1191).

\section{CONFLICTS OF INTEREST}

D Kim, DH Yoem, JH Ahn, WK You, and Sang Lee are employees of $\mathrm{ABL}$ Bio Inc., the company developing ABL001 (NOV1501/TR009). The other authors have no conflicts to declare.

\section{REFERENCES}

1. Russo AE and Strong VE (2019) Gastric cancer etiology and management in asia and the west. Annu Rev Med 70, 353-367

2. Sitarz R, Skierucha M, Mielko J, Offerhaus GJA, Maciejewski R and Polkowski WP (2018) Gastric cancer: epidemiology, prevention, classification, and treatment. Cancer Manag Res $10,239-248$

3. Kanat O, O'Neil B and Shahda S (2015) Targeted therapy for advanced gastric cancer: A review of current status and future prospects. World J Gastrointest Oncol 7, 401-410

4. Lin Y, Wu Z, Guo W and Li J (2015) Gene mutations in gastric cancer: a review of recent next-generation sequencing studies. Tumour Biol 36, 7385-7394

5. Guruharsha KG, Kankel MW and Artavanis-Tsakonas S 
(2012) The Notch signalling system: recent insights into the complexity of a conserved pathway. Nat Rev Genet $13,654-666$

6. Andersson ER, Sandberg R and Lendahl U (2011) Notch signaling: simplicity in design, versatility in function. Development 138, 3593-3612

7. Hori K, Sen A and Artavanis-Tsakonas S (2013) Notch signaling at a glance. J Cell Sci 126, 2135-2140

8. Bagheri V, Memar B, Behzadi R et al (2018) Isolation and identification of chemotherapy-enriched sphere-forming cells from a patient with gastric cancer. J Cell Physiol 233, 7036-7046

9. Ishigami S, Arigami T, Uenosono Y et al (2013) Clinical implications of DLL4 expression in gastric cancer. J Exp Clin Cancer Res 32, 46

10. Miao ZF, Xu H, Xu HM et al (2017) DLL4 overexpression increases gastric cancer stem/progenitor cell self-renewal ability and correlates with poor clinical outcome via Notch-1 signaling pathway activation. Cancer Med 6, 245-257

11. Aster JC, Pear WS and Blacklow SC (2017) The Varied Roles of Notch in Cancer. Annu Rev Pathol 12, 245-275

12. Lee D, Kim D, Choi YB et al (2016) Simultaneous blockade of VEGF and Dll4 by HD105, a bispecific antibody, inhibits tumor progression and angiogenesis. MAbs 8, 892-904

13. Bailly C (2019) Irinotecan: 25 years of cancer treatment. Pharmacol Res 148, 104398

14. Thuss-Patience PC, Kretzschmar A, Bichev D et al (2011) Survival advantage for irinotecan versus best supportive care as second-line chemotherapy in gastric cancer-a randomised phase III study of the Arbeitsgemeinschaft Internistische Onkologie (AIO). Eur J Cancer 47, 2306-2314

15. Pasquini G, Vasile E, Caparello C et al (2016) Third-line chemotherapy with irinotecan plus 5-fluorouracil in caucasian metastatic gastric cancer patients. Oncology 91, 311316

16. Seo MD, Lee KW, Lim JH et al (2008) Irinotecan combined with 5-fluorouracil and leucovorin as second-line chemotherapy for metastatic or relapsed gastric cancer. Jpn J Clin Oncol 38, 589-595

17. Sweeney TJ, Mailander V, Tucker AA et al (1999) Visualizing the kinetics of tumor-cell clearance in living animals. Proc Natl Acad Sci U S A 96, 12044-12049

18. den Dulk M, Verheij M, Cats A, Jansen EP, Hartgrink HH and Van de Velde CJ (2006) The essentials of locoregional control in the treatment of gastric cancer. Scand J Surg 95, 236-242

19. Reim D, Loos M, Vogl F et al (2013) Prognostic implications of the seventh edition of the international union against cancer classification for patients with gastric cancer: the Western experience of patients treated in a single-center European institution. J Clin Oncol 31, 263-271

20. Kim IH (2019) Current status of adjuvant chemotherapy for gastric cancer. World J Gastrointest Oncol 11, 679-685

21. Fujita K, Kubota $Y$, Ishida $H$ and Sasaki $Y$ (2015) Irinotecan, a key chemotherapeutic drug for metastatic colorectal cancer. World J Gastroenterol 21, 12234-12248

22. Chun JH, Kim HK, Lee JS et al (2004) Weekly irinotecan in patients with metastatic gastric cancer failing cisplatinbased chemotherapy. Jpn J Clin Oncol 34, 8-13

23. Yamao T, Shirao K, Matsumura Y et al (2001) Phase I-II study of irinotecan combined with mitomycin-C in patients with advanced gastric cancer. Ann Oncol 12, 1729-1735

24. Leslie JD, Ariza-McNaughton L, Bermange AL, McAdow R, Johnson SL and Lewis J (2007) Endothelial signalling by the Notch ligand Delta-like 4 restricts angiogenesis. Development 134, 839-844

25. Shutter JR, Scully S, Fan W et al (2000) Dll4, a novel Notch ligand expressed in arterial endothelium. Genes Dev 14, 1313-1318

26. Chatterjee S, Heukamp LC, Siobal M et al (2013) Tumor VEGF:VEGFR2 autocrine feed-forward loop triggers angiogenesis in lung cancer. J Clin Invest 123, 1732-1740

27. Fu L, Bu L, Yasuda T et al (2020) Gastric cancer stem cells: current insights into the immune microenvironment and therapeutic targets. Biomedicines 8, 7

28. Katoh $M$ and Katoh $M$ (2020) Precision medicine for human cancers with Notch signaling dysregulation (Review). Int J Mol Med 45, 279-297

29. Baek JH, Kim NJ, Song JK and Chun KH (2017) Kahweol inhibits lipid accumulation and induces Glucose-uptake through activation of AMP-activated protein kinase (AMPK). BMB Rep 50, 566-571

30. Kim NJ, Baek JH, Lee J, Kim H, Song JK and Chun KH (2019) A PDE1 inhibitor reduces adipogenesis in mice via regulation of lipolysis and adipogenic cell signaling. Exp Mol Med 51, 5

31. Cho Y, Kang HG, Kim SJ et al (2018) Post-translational modification of OCT4 in breast cancer tumorigenesis. Cell Death Differ 25, 1781-1795 